\title{
Rheological properties of aquaeous dispersion of styrene acrylate copolymer incorporating hollow microspheres and AEROSIL $®$
}

\author{
Natalia Saienko, ${ }^{1,}$, Dmitriy Demidov ${ }^{2}$, Yuri Popov ${ }^{1}$, Roman Bikov $^{1}$, \\ and Vyacheslav Butskyi ${ }^{1}$ \\ ${ }^{1}$ Kharkiv National University of Civil Engineering and Architecture, Sumska st. 40, 61002 Kharkiv, \\ Ukraine \\ ${ }^{2}$ Kharkiv State Auto-transport College, Constitution sq. 28, 61003 Kharkiv, Ukraine
}

\begin{abstract}
The rheological properties of aquaeous dispersion of styrene acrylate copolymer incorporating hollow aluminosilicate microspheres and AEROSIL ${ }^{\circledR} 300$ fumed silica (a high-range water reducing admixture) have been investigated using rotational viscometer. It is established that an increase in the degree of filling with hollow microspheres results in a significant increase in the viscosity of aqueous dispersion leading to the technological difficulties when coating the protected surfaces. AEROSIL ${ }^{\circledR} 300$ can be dosed in small amounts resulting in a decrease in the structural strength and optimal viscosity of aqueous dispersion of acrylate copolymer, which allows to significantly reduce the viscosity density, while maintaining relatively low water demand and characteristics of highly filled systems, which is crucial in the technology of their application. The combination of such fillers as AEROSIL ${ }^{\circledR} 300$ and hollow aluminosilicate microspheres in the styrene acrylate copolymer aqueous dispersion allows the combination of a number of positive effects: a reduction in the viscosity of the material during application (without dilution) by applying high shear rate and increasing viscosity (structuring) after application, and also during their storage and transportation.
\end{abstract}

\section{Introduction}

Waterborne coatings revealed environmentally friendly features, widely used for the production of protective and decorative coatings on nonmetallic substrates, but have recently been increasingly used in transport construction to protect metal and concrete from corrosion [1].

Such material provides an opportunity to exclude the toxic and fire-hazardous solvents, which are toxic to the environment when exposed to air during hardening. The formation technology and performance characteristics of coatings are determined by the properties of film-forming materials, which shall meet the following requirements: ensuring uniform thin-layer distribution on the surface of the substrate and forming of coatings with

\footnotetext{
* Corresponding author: natause@ukr.net
} 
predetermined functionality. Such requirements are fully consistent with waterborne coatings [2].

Due to the functional properties and relatively low cost, waterborne coatings based on acrylic binders have become the most widely used [3].

By rational combination of active ingredients: film-forming agent based on aqueous acrylate copolymer dispersion of the pigments, thickeners, targeted additives, mineral fillers and coatings with a given set of properties can be obtained.

Thus, incorporating of high-range water reducing admixtures (talc, kaolin, AEROSIL ${ }^{\circledR}$, titanium dioxide, a number of organic pigments) into aqueous dispersions of acrylates results in the formation of thixotropic coatings. Due to established structure thixotropic coatings do not flow, but are easily applied to the surface if this structure is damaged.

One of the most common fillers of this type is AEROSIL $\AA$, which also serves as a stabilizing agent of waterborne coatings, prevents the settling of pigments and imparts thixotropic properties of the coatings. This is a high-range water reducing admixture of amorphous silicon dioxide, obtained by high-temperature hydrolysis. The presence of silanol groups on the surface of the particles determines the hydrophilic nature of silica obtained according to the said method [4-8].

At present, hollow glass or ceramic microspheres are increasingly used to impart heatinsulating properties to coatings. Microspheres can be extracted or incorporating rarefied air (depending on the conditions of their production) and, thanks to a successful combination of spherical shape, controlled dimensions, low density, relatively high compressive strength; good heat and sound insulation; dielectric properties, are one of the promising technogenic fillers of waterborne coatings [9-12].

Therefore, the study of mineral filler effect on chemical composition of the rheological properties of highly-solid aqueous coatings remains a relevant applied research task, since the filling affects the structural characteristics and predetermines the coating technology respectively.

The objective of this paper was to study the influence of the chemical composition and specific surface of fillers on the acrylic aqueous dispersions properties, such as: optimal dynamic viscosity, structural and rheological properties

\section{Experimental program}

Acryl-styrene dispersion of ACRONAL ${ }^{\circledR} 290$ D, produced by BASF (non-volatile compounds content - $50 \% \mathrm{wt}$, PH 7.5...9.0, average particle size about $100 \mathrm{~nm}$, viscosity at $23{ }^{\circ} \mathrm{C}$ (ISO 3219, DIN 53019) at shear rate $-100 \mathrm{~s}^{-1}, 7 \ldots 15 \mathrm{mPa} \cdot \mathrm{s}$ ) was used as a paste film thickness.

As the modifiers of the dispersion of ACRONAL ${ }^{\circledR} 290$ D, cellulosic and acrylic thickeners, a defoamer without mineral oil on a polymer base and dispersing medium were used.

Hollow microspheres (MS) were used as fillers of water-dispersion paint materials (WDCM), which are finely dispersed, free-flowing powders consisting of thin-walled silica-alumina particles of spherical shape with a diameter of $10 \ldots 100 \mu \mathrm{m}$ and a specific surface area $0.61 \mathrm{~m}^{2} / \mathrm{g}$.

To regulate the rheological properties of WDCM, a filler AEROSIL $₫ 300$ (A-300) with a specific surface area $300 \mathrm{~m}^{2} / \mathrm{g}$ was used. The mineral composition of the fillers is presented in Table 1 .

Compositions of modifiers and mineral fillers for the study based on the dispersion of ACRONAL ${ }^{2} 290$ D, were prepared as follows: the components of the composition were dispersed for 20 minutes in a mixer in the form of a dissolver with a paddle stirrer at a rotational speed of $60 \ldots 120 \mathrm{rpm}$ until a homogeneous mass was obtained. 
Table 1. Compound mineral powders

\begin{tabular}{|c|c|c|c|c|c|c|c|c|c|}
\hline \multirow[b]{2}{*}{ Filler } & \multirow{2}{*}{$\begin{array}{c}\text { Surface } \\
\text { area, } \\
\mathbf{m}^{2} / \mathrm{g}\end{array}$} & \multirow{2}{*}{$\rho, \mathbf{g} / \mathbf{c m}^{3}$} & \multirow[b]{2}{*}{ pH } & \multicolumn{6}{|c|}{ Mineral composition, \% wt } \\
\hline & & & & $\mathrm{SiO}_{2}$ & $\mathbf{A l}_{2} \mathbf{O}_{3}$ & IgO & $\mathrm{Fe}_{2} \mathrm{O}_{3}$ & $\mathrm{TiO}_{2}$ & $\mathrm{CaO}$ \\
\hline MS & 0.61 & $0.58 \ldots 0.69$ & 8.5 & $\begin{array}{c}52.2 \ldots \\
64.3\end{array}$ & $\begin{array}{c}18.0 \ldots \\
39.0\end{array}$ & $\begin{array}{c}1.0 \ldots \\
2.0\end{array}$ & $\begin{array}{c}1.2 \ldots \\
10.0\end{array}$ & $\begin{array}{c}0.6 \ldots \\
1.0\end{array}$ & $\begin{array}{c}0.1 \ldots \\
5.8\end{array}$ \\
\hline A-300 & 300.00 & $0.05 \ldots 0.06$ & 4.5 & 99.9 & 0.05 & - & 0.003 & 0.03 & - \\
\hline
\end{tabular}

The rheological properties of the compositions were determined using a Reotest-2 rotary viscometer (Germany) with a cylinder-cylinder working assembly using a measuring cylinder $\mathrm{H}$. The flow curves of the compositions were recorded with a change in the speed from 0.1667 to $72.9 \mathrm{sec}^{-1}$ at temperatures: 286,296 and $306 \mathrm{~K}$. The sample of the prepared compositions was then temperature-controlled for 15 minutes.

To establish the fact that the compositions under study have thixotropic properties (form a hysteresis loop), the flow curves were also measured for forward and reverse travel, that is, for the rise and fall of the shear stress $(\tau)$, respectively. Prior to the test, the composition was subjected to a 10-minute deformation at the maximum shear rate $\left(72.9 \mathrm{~s}^{-1}\right)$ and a rest period of 10 minutes during the reverse course of the measurements.

\section{Results and discussion}

The findings of rheological tests of aqueous dispersion (WDCM) based on styrene acrylate copolymer ACRONAL ${ }^{\circledR} 290 \mathrm{D}$ are presented in the form of rheological flow curves (the dependence of the shear stress $\tau(\mathrm{Pa})$ on the fillers (initial) and upon the introduction of the AEROSIL ${ }^{\circledR}(A-300)$ fillers and hollow microspheres $)$ on the shear rate $\operatorname{Dr}\left(\mathrm{s}^{-1}\right)$ (Fig. 1a) and in the form of the viscosity change curves $\eta(\mathrm{Pa} \cdot \mathrm{s})$ versus the shear stress $\tau(\mathrm{Pa})$ (Fig. 1b).

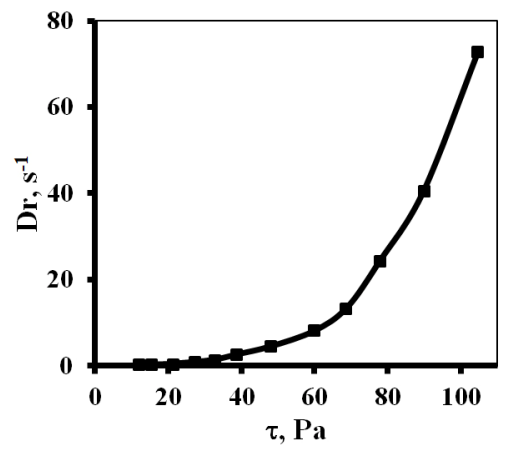

a)

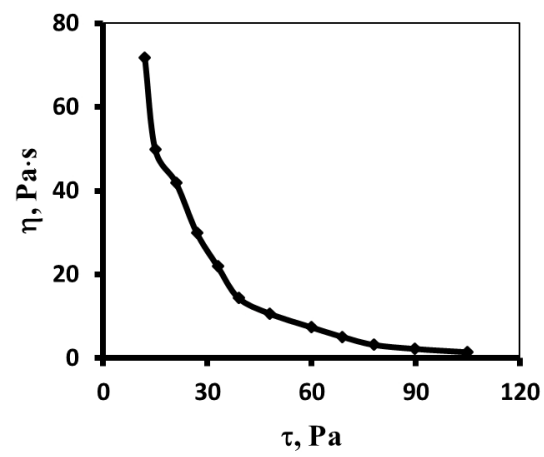

b)

Fig.1. Rheological dependences of the initial WDCM in the form of flow curves (a) and viscosity change curves on the shear stress (b).

First of all, the anomalous nature of the rheological curve (Fig. 1a) as a nonlinear plastic flow of the initial WDCM and the presence of a static limiting shear stress equal to $15.1 \mathrm{~Pa}$ (according to Rehbinder, the static static yield point $\mathrm{P}_{\mathrm{k} 1}$ [13]) is of primary interest. This indicates that the dispersed system has a highly developed spatial structure and its flow occurs under the condition that the shear stress value is comparable with the strength of this structure. Consequently, the conditional static yield strength is a measure of the strength of the structural framework. When passing through, the structural grid breaks down, which results in the presence of all nonlinear effects. 
The viscosity change in the narrow shear rate is observed on the viscosity change curve from the shear stress of the unfilled WDCM (Fig. 1b), which indicates a significant destruction of its spatial (coagulation) structure.

The character of the rheological curves shown in Fig. 1 testify that WDCM can be attributed to plastic bodies with nonlinear plastic behavior and ultimate shear stress. This conclusion is supported by the fact that rheological curves in the investigated range of shear stresses are well described by the well-known Herschel-Balkley equation [14]:

$$
\tau=\tau_{s}+\eta_{o p} \eta^{p}
$$

where $\tau_{s}$ is the conditional static yield point, $\mathrm{Pa} ; \eta_{o p}$ is the optimum water demand (OWD), $\mathrm{Pa} \cdot \mathrm{s} ; \gamma$ is the shear rate, $\mathrm{s}^{-1} ; n$ is the the exponent (flow index), which is a measure of the structuredness of systems with a nonlinear plastic pseudoplastic flow pattern $n<1$, and for Newtonian liquids $n=1$.

For the initial (unfilled) WDCM composition, the flow index is $0.355(\mathrm{n}<1)$, which indicates that it has a spatial structural grid that is destroyed by relatively small applied shear stresses, of the order of $15 \ldots 20 \mathrm{~Pa}$, and the coagulation structure is easily restored, which indicates the absence of hysteresis loops on the flow curves. It can be assumed that the main contribution to the process of structuring the unfilled WDCM is the interaction between the globules of the latexastirol-acrylate copolymer.

It is known that the introduction of fillers in the polymer dispersion leads to the appearance of a wide range of interactions (from weak physical forces to stronger hydrogen) arising at the polymer-filler-filler-filler interface resulting in the formation of coagulation structures. In structures of the coagulation type, the particles are connected by the intermolecular forces of Van der Waals-London, coagulation and point contacts arise between them. Such structures have a small strength, plastic, elastic and thixotropic properties [15]. The plastic flow is associated with thixotropic phenomena occurring in highly-solid materials. Often, the manifestation of structural viscosity is considered as a positive quality, as the coatings acquire a pasty consistency and in such systems pigments do not settle, materials can be applied thick layers on vertical surfaces without streaks.

Figure 2 shows the rheological flow curves of the WDCM, with a loading level of the MS of $20 \% \mathrm{wt}$ and $40 \% \mathrm{wt}$. As can be seen, the introduction of the microcircuit of the flow does not change, but a sharp increase in the strength of the structural grid occurs, as evidenced by the values of the conditional static yield point $\tau_{s}$, value, which increases more than 3...4 times (see Table 2).

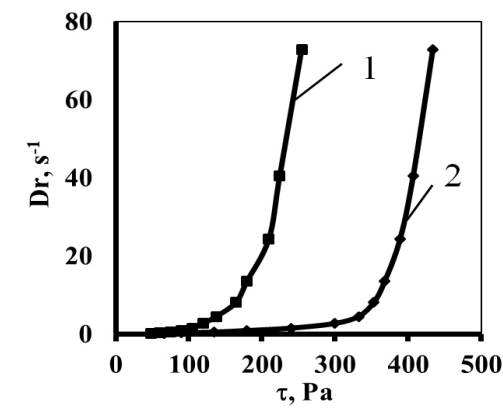

a)

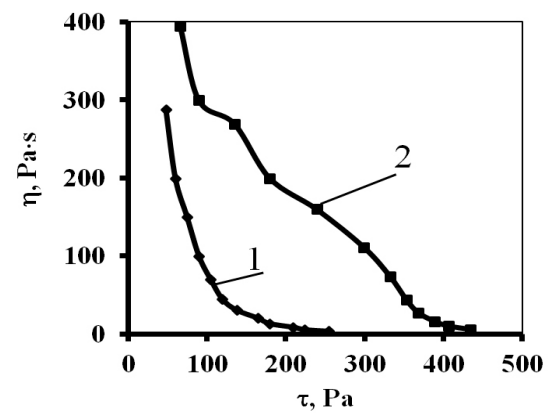

b)

Fig.2. Rheological dependences of WDCM incorporating MS of 20 (1) and 40\% (2) by mass in the form of flow curves (a) and viscosity change curves on shear stress (b). 
In Fig. $2 b$ shows the dependence of the optimum water demand (OWD) of microspherefilled WDCM dispersions on the shear stress. It can be seen that the initial values of the optimum water demand (OWD) $\eta_{0}$ increase from $89.8 \mathrm{~Pa} \cdot \mathrm{s}$ to $287.3 \mathrm{~Pa} \cdot \mathrm{s}$ with an MS content of $20 \% \mathrm{wt}$ and up to $395.1 \mathrm{~Pa} \cdot \mathrm{s}$ at $40 \% \mathrm{wt}$ (see Table 2 ).

The data presented indicate an increase in the energy between the partial interaction in the resulting structure and the number of contacts with an increase in the content of microspheres, as well as a decrease in the thickness of the interlayers of the dispersion medium. Important in our opinion is that the acidity index of the water extract of the microspheres themselves is $\mathrm{pH} 8.5$ and they, apparently, additionally contribute to the thickening of the styrene acrylate copolymer dispersion due to an increase in the degree of ionization of the carboxyl groups [16]. In addition, for microsphere-filled dispersions of the copolymer, there is also a significant decrease in the optimum water demand (OWD) at the initial sections of the deformation at low shear stresses, which is caused by the rupture at the initial stage of less strong contacts between dispersion globules, between mineral particles and polymeric globules, and also the orientation of partially destroyed aggregates in the direction of shear. Despite the considerable hardening of the structure incorporating WDCM microspheres, the coagulation structure is easily reconstructed camping, as evidenced by the absence of flow curves of the hysteresis loop.

The specificity of the interaction of AEROSIL ${ }^{\circledR}$ with aquaeous dispersion of the polymer lies in the fact that a relatively low mass fraction of the solid phase creates an anomalously high concentration of particles $-10^{15}-10^{16}$ per $\mathrm{cm}^{3}$ of the dispersion volume [17]. Thus, the investigated AEROSIL $®$ dispersions can be attributed to the so-called highly concentrated dispersive systems, for which the influence of the concentration factor on the properties is decisive.

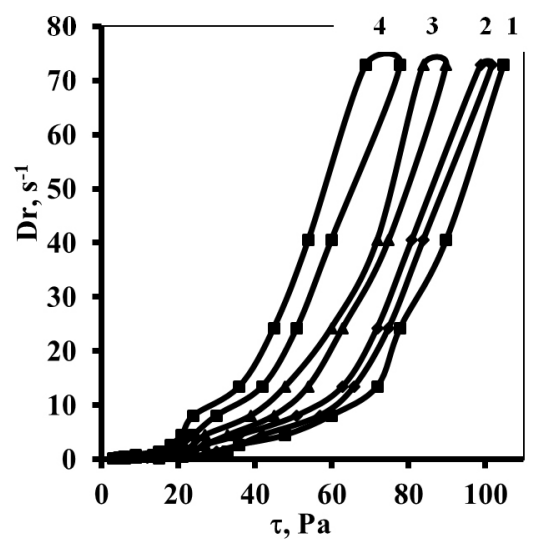

a)

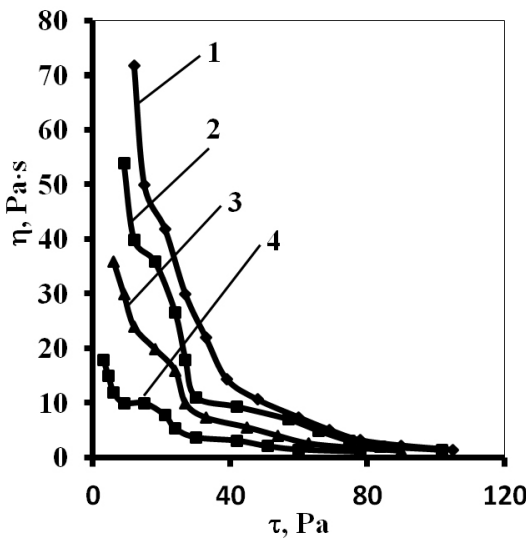

b)

Fig. 3. Rheological dependencies of WDCM A-300 content in the form of flow curves (a) and viscosity change curves versus shear stress (b): 1 - WDCM; 2 - A-300 $0.5 \%$ wt; 3 - A-300 $1.0 \%$ wt; 4 - A-300 $1.5 \%$ wt.

In Fig. 3 a, rheology-impenetrable dispersions of WDCM incorporating AEROSIL $®$ A-300 in an amount of $0.5,1.0$ and $1.5 \%$ by weight are presented. It can be seen that the nature of the flow curves does not change with the introduction of A-300, but the strength of the structural grid decreases, as evidenced by the values of the conditional static yield point, the value of which decreases from 15.1 Pa for WDCM to 6.1, 5.4 and 3.1 $\mathrm{Pa}$ at the introduction of A-300 in an amount of $0.5,1.0$ and $1.5 \% \mathrm{wt}$, Respectively (see Table 2). At the same time, the values of the initial optimum water demand (OWD) $\eta_{0}$ decrease by more than 2...4 times (see Table 2). This is most likely due to the fact that when the A-300 is 
introduced, the alkalinity of the WDCM itself decreases (the $\mathrm{pH}$ value of aquaeous extract of AEROSIL $\AA$ is $\mathrm{pH}=4.1)$ resulting in a decrease in the viscosity of WDCM due to a decrease in the degree of ionization of carboxyl groups $(-\mathrm{COOH})$ particles of latex.

The analysis of the obtained results shows that the rheological behavior of aquaeous acrylic-styrene dispersions incorporating AEROSIL ${ }^{\circledR}$ has all the characteristics typical for highly concentrated disperse systems with a coagulation-type structure. A sign of this is the nonlinear character of rheological curves and incomplete display of the rheological behavior of dispersions under dynamic conditions. This is manifested in the appearance of a hysteresis loop, whose dimensions increase noticeably with increasing AEROSIL $®$ content, and the rheological curves themselves are shifted to the region of lower shear stresses.

At the same time, enhancement of packing density hollow microspheres up to $40 \% \mathrm{wt}$ Significantly increases the viscosity of the composition (by 4 times) resulting in technological difficulties in the application of heat-insulating coatings to the protected surfaces. These difficulties are associated with the need to avoid the flow of the film in the gravitational field. Such a "flow" of the material can lead to the appearance of an inhomogeneity in its film thickness. Therefore, considering the fact that the introduction of A-300 leads to a certain decrease in the structural strength and optimum water demand (OWD) of aquaeous dispersion of the acrylate copolymer, the rheological properties of WDCM filled simultaneously with hollow microspheres and A-300 were studied.

In Fig. 3a, the flow curves of WDCM incorporating hollow microspheres (40\% wt) and additionally with AEROSIL ${ }^{\circledR}(0.5,1.0$ and $1.5 \% \mathrm{wt})$ are presented. It can be seen that the nature of the flow curves does not change with the addition of the A-300 to the filled system, but the strength of the structural grid decreases, as evidenced by the values of the conditional static yield point $\left(\tau_{s}\right)$, which decreases from $65.8 \mathrm{~Pa}$ to $59.9,52.4$ and $44.9 \mathrm{~Pa}$ with the introduction of A-300 in an amount of 0.5, 1.0 and $1.5 \% \mathrm{wt}$, respectively (see Table 2). At the same time, the values of the initial optimum water demand (OWD) $\left(\eta_{0}\right)$ are reduced from 395.1 Pa.s to 359.1, 314.2 and 269.3 Pa.s (see Table 2), and the shear stress interval of the WDCM, filled simultaneously with microspheres and AEROSIL $®$. This system is also characterized by the nonlinear behavior of rheological curves and the appearance of hysteresis loops, indicating an incomplete reconstruction of the structure of the complexly filled WDCM in a dynamic mode with forward and reverse deformation of shear stresses.

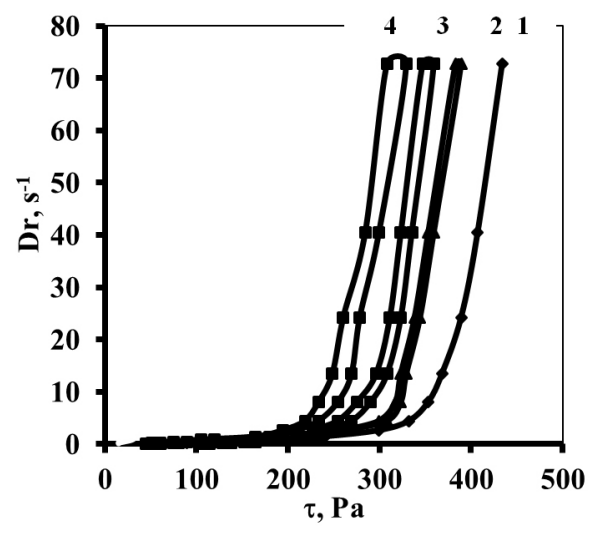

a)

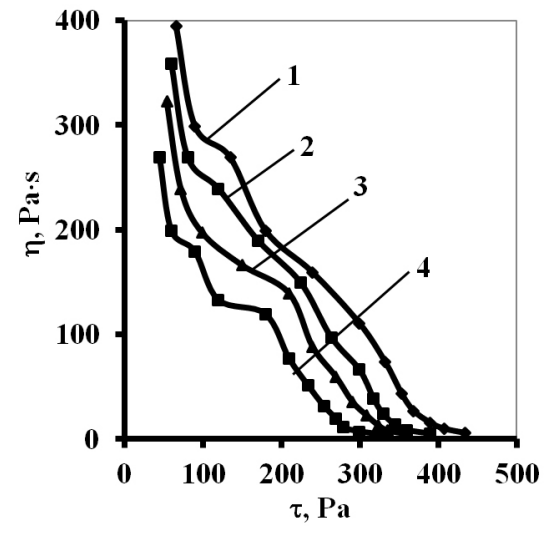

b)

Fig. 4. Rheological dependences of WDCM on the content of MS and A-300 in the form of flow curves (a) and viscosity change curves on shear stress (b): 1 - MS $40 \% \mathrm{wt} ; 2$ - MS 40+A-300 $0.5 \%$ wt; 3 - MS 40+A-300 1.0\% wt; 4 - MS 40+A-300 1.5\% wt. 
To assess the degree of structuring and the strength of intermolecular interactions in the WCDMA, structural and rheological properties allow for structural and rheological properties. Table 2 shows the structural and rheological properties: the conditionally static yield strength $\left(\tau_{s}\right)$, the viscosity at the minimum rate, the onset of failure (initial optimum water demand (OWD) $\left(\eta_{0}\right)$, the minimum viscosity at the maximum strain rate $\left(\eta_{\min }\right)$, and $n$ - the exponent in the Herschel-Balkley equation.

Table 2. Structuraal and rgeological characteristics.

\begin{tabular}{|l|c|c|c|c|}
\hline \multicolumn{1}{|c|}{ Compositions, \% wt } & $\boldsymbol{\tau}_{\boldsymbol{s}}, \mathbf{P a}$ & $\boldsymbol{\eta}_{\boldsymbol{0}}, \mathbf{P a} \cdot \mathbf{s}$ & $\boldsymbol{\eta}_{\boldsymbol{m i n}}, \mathbf{P a} \cdot \mathbf{s}$ & $\boldsymbol{n}$ \\
\hline WDCM & 15.1 & 89.8 & 5.3 & 0.355 \\
\hline WDCM+A-300 (0.5) & 6.1 & 35.9 & 4.9 & 0.393 \\
\hline WDCM+A-300 (1.0) & 5.4 & 32.3 & 4.1 & 0.433 \\
\hline WDCM+A-300 (1.5) & 3.1 & 18.1 & 3.1 & 0.534 \\
\hline WDCM+MC (20) & 47.9 & 287.3 & 13.3 & 0.269 \\
\hline WDCM+MC (40) & 65.8 & 395.1 & 27.3 & 0.296 \\
\hline WDCM+MC (40)+A-300 (0.5) & 59.9 & 359.1 & 24.4 & 0.292 \\
\hline WDCM+MC (40)+A-300 (1.0) & 52.4 & 314.2 & 22.8 & 0.303 \\
\hline WDCM+MC (40)+A-300 (1.5) & 44.9 & 269.3 & 19.9 & 0.315 \\
\hline
\end{tabular}

\section{Conclusions}

The experimental studies on the rheological properties of aquaeous dispersion of styrene acrylate copolymer incorporating aluminosilicate microspheres (MS) and high-range water reducing AEROSIL $₫ 300$ fumed silica (A-300) determined the viscosity and strain rate of WDCM versus the shear stress for the fillers: MC from 20 up to $40 \%$ wt and A-300 from 0.5 to $1.5 \%$ wt.

By the nature of the rheological curves, the unfilled WDCM refers to plastic bodies with nonlinear plastic behavior and limiting shear stress, has a spatial structural grid that is destroyed by relatively small applied shear stresses, of the order of 15-20 Pa and easily restores the coagulation structure, as evidenced by the absence on the curves of the flow of the hysteresis loop.

The degree of thixotropy of the WDCM incorporating A-300 is characterized by the area of hysteresis loop and the absolute shear stress at low shear rate and increases with the content of the filler, since the coagulation interaction of the particles has a major role in the formation of spatial structures. The introduction of small additives A-300 (0.5 ..1.5\% wt) results in a certain decrease in the structural strength and optimum water demand (OWD) of aquaeous dispersion of the acrylate copolymer, which allows to significantly reduce the viscosity and regulate the rheological properties of the WCDMA application.

Enhancement of packing density of hollow microspheres up to $40 \%$ wt significantly increases the viscosity of the composition resulting in technological difficulties when applying heat-insulating coatings to the protected surfaces. These difficulties are associated with the need to avoid the flow of the film in the gravitational field. Such a "flow" of the material can lead to the appearance of an inhomogeneity in its film thickness.

Incorporating of AEROSIL® to WCDMA allows to regulate the rheological properties at the stage of production of coatings or before using them. That allows to combine a number of positive effects simultaneously: decrease in the viscosity of the material during application (without dilution) due to application of high shear rate and viscosity increase (structuring) after application, as well as during storage and transportation. 


\section{References}

1 V. Lobkovsky, Industrial coatings, 4, 28-31 (2016)

2 E.E. Kazakova, Water-dispersions acrylic paint and varnish materials for construction purposes, (Moscow, 2003)

3 N. Saienko, D. Demidov, M. Myagkikh, Scientific Bulletin of Civil Engineering, 86 (4), 154-157 (2016)

4 G. Michael, Russian Coatings Journal, 6, $42-47$ (2007)

5 M. Dolz, F. González, J. Delegido, M. Hernández and J. Pellicer, Journal of pharmaceutical sciences, 89 (6), 790-797 (2000)

6 N. Saienko, R. Bukov, D. Demidov and D. Kovalenko, International Scientific Conference "Transport technologies and infrastructure", 356-358 (2018)

7 A. S. Makarov, I. A. Andreeva, V. Y. Tretinnik, Colloid Journal, 63 (6), 731-737 (2001)

8 V. Katnov, S. Stepin, R. Katnova, R. Mingaliyeva and P. Grishin, Herald of Kazan Technological University, 15, (7), 95-96 (2012)

9 H. S. Katz, J.V. Milewski, Handbook of fillers and reinforcements for plastics, (New York: Van Nostrand Reinhold Co, 1978)

10 L. Vakhitova, A. Zavertatnyy, F+ S: fare and security, 45 (3), 64-66 (2010)

11 A. Inozemtsev, Ye. Korolev, Industrial and Civil Engineering, 10, 80-83 (2013)

12 V.S. Drozhzhin, I.V. Piculin, J. Moscow Phys. Soc., 9, 209-214 (1999)

13 P. V. Mikhailov, P.A. Rebinder, Colloid Journal, 18 (2), 107-119 (1955)

14 V.N. Matveenko, Kirsanov E.A. MSU Vestnik, Series 2. Chemistry, 52 (4), 243-276 (2011)

15 A.Y. Malkin A. Y. Rheology in the processes of formation and transformation of polymers, Moscow, (1985)

16 V.A. Epishkina, R.N. Celms, V.K. Vasiliev and A.M. Kiselev, Proceedings of high schools. Technology of textile industry, 6, 86-92 (2006)

17 V. Zakordonsky, B. Lazaruk, Visnyk of tne Lviv University. Series Chemistry, 55 (2), 472-485 (2014) 